Games and Economic Behavior v. 42(2) Feb. 2003, pp. 307-314

\title{
Strong Comparative Statics of Equilibria *
}

\author{
Federico Echenique ${ }^{\dagger} \quad$ Tarun Sabarwal ${ }^{\ddagger}$
}

March 19, 2002

Running title: Strong Comparative Statics of Equilibria

${ }^{*}$ We are deeply grateful to Ilya Segal and Chris Shannon for their detailed suggestions and to Laura Gascue for her help with programming. This work was completed in February 2000 while we were both graduate students at the University of California at Berkeley. The opinions in this paper are those of the authors and do not necessarily reflect those of the Board of Governors or its staff.

†Universidad Torcuato Di Tella, and Facultad de Ciencias Sociales, Universidad de la República

$\ddagger$ (corresponding author) Mail Stop 93, Federal Reserve Board, Washington DC 20551, USA, email: tarun.sabarwal@frb.gov, Fax: (202) 7285887 


\begin{abstract}
Some results in the monotone comparative statics literature tell us that if a parameter increases, some old equilibria are smaller than some new equilibria. We give a sufficient condition such that at a new parameter value every old equilibrium is smaller than every new equilibrium. We also adapt a standard algorithm to compute a minimal such newer parameter value and apply this algorithm to a game of network externalities. Our results are independent of a theory of equilibrium selection and are valid for games of strategic complementarities.
\end{abstract}

JEL Classification: C61, C62, C63, C72

Keywords: Comparative statics, computing equilibria, strategic complementarities, supermodular games. 


\section{Introduction}

In a parameterized model we often want to know how an equilibrium changes if we change the parameter. If the parameter space is a partially ordered set and the state space or the decision space is also a partially ordered set, we often want to know when raising the parameter increases the equilibrium. In economics, some models in this framework arise as games of strategic complementarities (as defined in Milgrom and Shannon (1994)). These games include supermodular games (as defined in Topkis (1979), in Milgrom and Roberts (1990) and in Vives (1990)). Classes of such games are games of coordination (for example, games of macroeconomic coordination failure and games of network externalities) and games of industry behavior (for example, games of Bertrand Oligopoly when the products of the firms are substitutes). For other examples, see Milgrom and Roberts (1990), Topkis (1998), and Vives (1999).

For these types of models, some of the results in the recent literature on monotone comparative statics give us conditions from which we can conclude that if a parameter increases, some old equilibria are smaller than some new equilibria. For example, Lippman, Mamer, and McCardle (1987) show that there is an old equilibrium that is smaller than some new equilibrium. In a more general framework, Milgrom and Roberts (1990) and Sobel (1988) show that the smallest old equilibrium is smaller than the smallest new equilibrium and the largest old equilibrium is smaller than the largest new equilibrium. Milgrom and Shannon (1994) prove the same result in an even more general framework. But we may not be able to say that a given equilibrium at a lower parameter value is smaller than another given equilibrium at a higher parameter value. In the absence of a widely accepted theory of equilibrium selection, one way to unambiguously determine that an old equilibrium is smaller than a new equilibrium is if every old equilibrium is smaller than every new equilibrium. In this paper, we give a sufficient condition which, when satisfied at a higher parameter value, allows us to conclude that every old equilibrium is smaller than every new equilibrium. We also give an algorithm to compute 
a minimal such higher parameter value and as an example, apply this algorithm to a game of network externalities. Our results are independent of any theory of equilibrium selection and apply to games of strategic complementarities.

In the next section, we formalize the concepts and prove the results mentioned above. In the last section, we give an application of the algorithm for a game of network externalities.

\section{Model and Results}

In this section, we first formalize a notion of a parameterized model and an equilibrium in it. Then we provide a sufficient condition to conclude that at a higher parameter value, every old equilibrium is smaller than every new equilibrium. Finally, we provide an algorithm to compute an approximation to a minimal such higher parameter value.

The notion of a parameterized model that we use is formalized in the definition of an increasing family of correspondences given below. For a given parameter value, an equilibrium in this model is a fixed point of the section of the correspondence determined by the given parameter value.

Let $X$ be a partially ordered set and $A, B$ be two subsets of $X$. We shall say that $A$ is weakly smaller than $B$ if for every $a \in A$ there is $b \in B$ with $a \preceq b$ and for every $b \in B$ there is $a \in A$ with $a \preceq b$. A correspondence $\phi: X \rightarrow X$ is weakly increasing if for every $x, x^{\prime} \in X, x \prec x^{\prime}$ implies that $\phi(x)$ is weakly smaller than $\phi\left(x^{\prime}\right)$. We shall say that $A$ is strongly smaller than $B$ if for every $a \in A$ and every $b \in B, a \preceq b$. Notice that if $A$ has a largest element $\bar{a}$ and $B$ has a smallest element $\underline{b}$ then $A$ is strongly smaller than $B$ if and only if $\bar{a} \preceq \underline{b}$.

For the sake of completeness, let us also define some preliminary concepts. A detailed discussion of these concepts can be found in Topkis (1998). A partially ordered set $X$ is a lattice if whenever $x, y \in X$, both $x \wedge y=\inf \{x, y\}$ and $x \vee y=\sup \{x, y\}$ exist in $X$. It is complete if for every nonempty subset $A$ of $X, \inf A, \sup A$ exist in $X$. A 
nonempty subset $A$ of $X$ is a sublattice if for all $x, y \in A, x \wedge_{X} y, x \vee_{X} y \in A$, where $x \wedge_{X} y$ and $x \vee_{X} y$ are obtained taking the infimum and supremum as elements of $X$ (as opposed to using the relative order on $A$ ). A nonempty subset $A \subset X$ is subcomplete if $B \subset A, B \neq \emptyset$ implies $\inf _{X} B$, $\sup _{X} B \in A$, again taking inf and sup of $B$ as a subset of $X$.

Now, suppose $X$ is a complete lattice and $T$ a partially ordered set. An increasing family of correspondences, denoted $\left(\phi_{t}: t \in T\right)$, is a correspondence $\phi: X \times T \rightarrow X$ such that (1) for every $t$, the correspondence $x \mapsto \phi_{t}(x)$ is weakly increasing, upper hemicontinuous and subcomplete sublattice valued and (2) for every $x$, the correspondence $t \mapsto \phi_{t}(x)$ is weakly increasing. For each $t$, the equilibrium set at $t$ is $\mathcal{E}(t)=\left\{x \in X: x \in \phi_{t}(x)\right\}$, the set of fixed points of $\phi_{t}$. For example, in games of strategic complementarities, the product of the best response correspondences of the players is an increasing family of correspondences and for a given parameter value, the set of Nash equilibria in the game is the set of fixed points of the section of the correspondence determined by this parameter value. (See Lemma 4.2.2 in Topkis (1998) and Lemma 1 in Echenique (2002).)

For a given parameter value, we are interested in higher parameter values such that the equilibrium set at the given parameter value is strongly smaller than the equilibrium set at the higher parameter value. For notational convenience, we use the following definition for these higher parameter values. Let $\left(\phi_{t}: t \in T\right)$ be an increasing family of correspondences and $t_{0} \in T$. A $\hat{t} \in T$ with $t_{0} \preceq \hat{t}$ is large enough for $t_{0}$ if $\mathcal{E}\left(t_{0}\right)$ is strongly smaller than $\mathcal{E}(\hat{t})$. As, for every $t$, the equilibrium set is a complete lattice (by Theorem 2.5.1 in Topkis (1998), for each $t, \mathcal{E}(t)$ is a complete lattice), $\mathcal{E}\left(t_{0}\right)$ is strongly smaller than $\mathcal{E}(\hat{t})$ if and only if $\sup \mathcal{E}\left(t_{0}\right) \preceq \inf \mathcal{E}(\hat{t})$. The following theorem tells us when a $\hat{t}$ is large enough for $t_{0}$.

Theorem. Let $\left(\phi_{t}: t \in T\right)$ be an increasing family of correspondences, $t_{0} \in T, \underline{e}=$ $\inf \mathcal{E}\left(t_{0}\right)$ and $\bar{e}=\sup \mathcal{E}\left(t_{0}\right)$. For every $\hat{t} \in T$ with $t_{0} \preceq \hat{t}$, if $\bar{e} \preceq \inf \phi_{\hat{t}}(\underline{e})$ then $\hat{t}$ is large 
enough for $t_{0}$. Also, if $\hat{t}$ is large enough for $t_{0}$ then so is every $t^{\prime} \in T$ such that $\hat{t} \preceq t^{\prime}$.

Proof: Using a slightly generalized version of Theorem 2.5.2 in Topkis (1998) - see the lemma in the appendix of this paper — we can say that if $t_{0} \preceq \hat{t}$ then $\underline{e} \preceq \inf \mathcal{E}(\hat{t})$. As the correspondence $x \mapsto \phi_{\hat{t}}(x)$ is weakly increasing, we have $\inf \phi_{\hat{t}}(\underline{e}) \preceq \inf \phi_{\hat{t}}(\inf \mathcal{E}(\hat{t}))$. As $\mathcal{E}(\hat{t})$ is a complete lattice, we have $\inf \mathcal{E}(\hat{t}) \in \phi_{\hat{t}}(\inf \mathcal{E}(\hat{t}))$ and therefore, $\inf \phi_{\hat{t}}(\inf \mathcal{E}(\hat{t})) \preceq$ $\inf \mathcal{E}(\hat{t})$. We conclude that if $\bar{e} \preceq \inf \phi_{\hat{t}}(\underline{e})$ then $\bar{e} \preceq \inf \phi_{\hat{t}}(\inf \mathcal{E}(\hat{t})) \preceq \inf \mathcal{E}(\hat{t})$ so that $\hat{t}$ is large enough for $t_{0}$. Applying the lemma in the appendix once more, we conclude that for every $t^{\prime} \in T$, if $\hat{t} \preceq t^{\prime}$ then $\sup \mathcal{E}\left(t_{0}\right) \preceq \inf \mathcal{E}(\hat{t}) \preceq \inf \mathcal{E}\left(t^{\prime}\right)$ so that $t^{\prime}$ is large enough for $t_{0}$.

\section{Insert Figure 1}

In Figure 1, we highlight the condition given in this theorem. Figure 1 has three sections of an increasing family of correspondences, determined at $t_{0} \preceq t^{\prime} \preceq \hat{t}$. These sections are singleton-valued. The equilibrium set at $t_{0}$ consists of three points where $\phi_{t_{0}}$ intersects the diagonal. The smallest equilibrium is labeled $\underline{e}$ and the largest equilibrium is labeled $\bar{e}$. From the figure we can see that the condition in the theorem is satisfied for $\hat{t}$ so that $\hat{t}$ is large enough for $t_{0}$ whereas it is not satisfied for $t^{\prime}$ and $t^{\prime}$ is not large enough for $t_{0}$.

In the remainder of this paper, we present a computational alternative to the theorem that helps us compute a $\hat{t}$ large enough for $t_{0}$. When $T$ is an order interval, and this is the case on which we shall focus in the rest of this paper, there is a distinguished value such that for each given value, if this distinguished value is not large enough for the given value then there is no point in $T$ that is large enough for the given value. Formally, suppose $T=[\underline{t}, \bar{t}]=\{t \in T \mid \underline{t} \preceq t \preceq \bar{t}\},\left(\phi_{t}: t \in T\right)$ is an increasing family of correspondences and $t_{0} \in T$. Then it is obvious that there exists a $\hat{t} \in T$ large enough for $t_{0}$ if and only if $\bar{t}$ is large enough for $t_{0} .{ }^{1}$ Therefore, in this case, to determine if there is a point in $T$

\footnotetext{
${ }^{1}$ It is easy to generalize this condition to the case where $T$ is an arbitrary partially ordered set with $\sup T \in T$. In this case, there exists $\hat{t} \in T$ large enough for $t_{0}$ if and only if $\sup T$ is large enough for $t_{0}$.
} 
large enough for $t_{0}$ we need only determine if the largest point in $T$ is large enough for $t_{0}$. We can do this by using the following algorithm.

Algorithm 1. (Topkis (1979)) Let $\left(\phi_{t}: t \in T\right)$ be an increasing family of correspondences and $\hat{t} \in T$. Fix $x \in X$.

1. Set $k=0$ and $x_{0}=x$.

2. If $\phi_{\hat{t}}\left(x_{k}\right)$ has a smallest element set $x_{k+1}=\inf \phi_{\hat{t}}\left(x_{k}\right)$ and go to next step. Else stop.

3. Set $k=k+1$. Return to step 2 and continue.

From Theorem 4.3.3 in Topkis (1998) we know that if $\left(\phi_{t}: t \in T\right)$ is an increasing family of correspondences, $\hat{t} \in T, x=\inf X$ then the sequence $\left(x_{k}\right)$ generated by Algorithm 1 converges to $\inf \mathcal{E}(\hat{t})$. Also, if we let $\hat{t}=t_{0}, x=\sup X$ and in step 2 of Algorithm 1 change the word smallest to largest and inf to sup then the sequence generated by this modified algorithm converges to $\sup \mathcal{E}\left(t_{0}\right)$. Thus, given two points $t_{0}$ and $\hat{t}$ in $T$ with $t_{0} \preceq \hat{t}$ we can determine whether $\hat{t}$ is large enough for $t_{0}$ or not. In particular, when $T=[\underline{t}, \bar{t}]$ we can determine if there is a point in $T$ large enough for $t_{0}$ by determining if $\bar{t}$ is large enough for $t_{0}$.

Once we know that $\bar{t}$ is large enough for $t_{0}$, we can invoke any of a number of algorithms to determine a minimal $\hat{t}$ that is large enough for $t_{0}$. We present one such algorithm. Our algorithm is slightly different from one that invokes Algorithm 1 in a standard way (that is, by using Theorem 4.3.3 in Topkis (1998)). We use some of the information computed in earlier iterations whereas applying Algorithm 1 in a standard way does not. Elementary versions of our algorithm occur frequently in numerical analysis. For example see Section 3.4 in Press, Flannery, Teukolsky, and Vetterling (1989).

Algorithm 2. Let $\left(\phi_{t}: t \in T\right)$ be an increasing family of correspondences and $t_{0} \in T=$ $[\underline{t}, \bar{t}] \subset \Re^{m}$. Using Algorithm 1, set $\underline{e}=\inf \mathcal{E}\left(t_{0}\right)$ and $\bar{e}=\sup \mathcal{E}\left(t_{0}\right)$. Fix a convergence criterion, $\epsilon>0$. Let $K \geq 1$ be such that $\frac{1}{2^{K}} \leq \epsilon$. 
1. Set $k=0, \underline{t}_{0}=t_{0}, \bar{t}_{0}=\bar{t}$ and $\underline{e}_{0}=\underline{e}$.

2. Set $\hat{t}_{k+1}=\underline{t}_{k}+\frac{1}{2}\left(\bar{t}_{k}-\underline{t}_{k}\right)$.

3. Using Algorithm 1 with $\hat{t}=\hat{t}_{k+1}$ and $x=\underline{e}_{k}$, set $\underline{e}_{k+1}=\inf \mathcal{E}\left(\hat{t}_{k+1}\right)$.

4. If $\bar{e} \preceq \underline{e}_{k+1}$ set $\underline{t}_{k+1}=\underline{t}_{k}, \bar{t}_{k+1}=\hat{t}_{k+1}$ and $\underline{e}_{k+1}=\underline{e}_{k}$ and go to next step. Else set $\underline{t}_{k+1}=\hat{t}_{k+1}$ and $\bar{t}_{k+1}=\bar{t}_{k}$ and go to next step.

5. If $k \leq K-1$ set $k=k+1$, return to step 2 and continue. Else stop.

This algorithm searches for the smallest point in the line segment from $t_{0}$ to $\bar{t}$ that is large enough for $t_{0}{ }^{2}$ It does this by dividing this line segment into halves and using Algorithm 1, testing whether the highest point in the lower half is large enough for $t_{0}$ or not. If it is then the algorithm changes the starting point in Algorithm 1 and proceeds to divide the lower half into halves and repeats the loop. Otherwise it does not change the starting point in Algorithm 1, divides the upper half into halves and repeats the loop.

In this algorithm a standard way to invoke Algorithm 1 is to use Theorem 4.3.3 in Topkis (1998) and always start Algorithm 1 from $x=\inf X$. This does not use information computed in earlier iterations. We use this information by starting Algorithm 1 at an earlier minimal fixed point. This can make our algorithm better than the one constructed in a standard way.

It is obvious that the sequences $\left(\underline{t}_{k}\right)$ and $\left(\bar{t}_{k}\right)$ generated by this algorithm are respectively, weakly increasing and weakly decreasing. It is easy to see that for every $k, \underline{t}_{k} \leq \bar{t}_{k}$ and $\bar{t}_{k+1}-\underline{t}_{k+1}=\frac{1}{2}\left(\bar{t}_{k}-\underline{t}_{k}\right)$ (and hence $\left.\bar{t}_{k}-\underline{t}_{k}=\frac{1}{2^{k}}\left(\bar{t}-t_{0}\right)\right)$. It is also easy to see that if $\bar{t}$ is large enough for $t_{0}$ then for every $k, \bar{t}_{k}$ is large enough for $t_{0}$ and if $t_{0}$ is not large enough for $t_{0}$ then for every $k, \underline{t}_{k}$ is not large enough for $t_{0}$. Each of these statements can be proved easily using induction.

\footnotetext{
${ }^{2}$ The line segment from $t_{0}$ to $\bar{t}$ is the convex hull of these two points.
} 
Recall that if $\bar{t}$ is not large enough for $t_{0}$ then there is no point in $T$ that is large enough for $t_{0}$. Also, if $t_{0}$ is large enough for $t_{0}{ }^{3}$ then $t_{0}$ is a minimal parameter value large enough for $t_{0}$. When either of these conditions hold, we don't need the algorithm given above. When neither of these conditions holds, we have the following proposition.

Proposition. Let $\left(\phi_{t}: t \in T\right)$ be an increasing family of correspondences and $t_{0} \in T=$ $[\underline{t}, \bar{t}] \subset \Re^{m}$. Suppose $\bar{t}$ is large enough for $t_{0}$ and $t_{0}$ is not large enough for $t_{0}$. Then, (1) For every $\epsilon>0$, there is $\hat{t} \in T$ such that $\hat{t}$ is large enough for $t_{0}$ and $\hat{t}-\epsilon\left(\bar{t}-t_{0}\right)$ is not large enough for $t_{0}$ and

(2) The sequences generated by Algorithm 2 converge to the infimum of those points in the line segment from $t_{0}$ to $\bar{t}$ that are large enough for $t_{0}$.

Proof: To prove the first statement fix $\epsilon>0$, let $K, \bar{t}_{K}$ and $\underline{t}_{K}$ be as in the previous algorithm and let $\hat{t}=\bar{t}_{K}$. We know that $\bar{t}_{K}$ is large enough for $t_{0}$ and $\underline{t}_{K}$ is not large enough for $t_{0}$. Also, $\hat{t}-\epsilon\left(\bar{t}-t_{0}\right) \leq \bar{t}_{K}-\frac{1}{2^{K}}\left(\bar{t}-t_{0}\right)=\underline{t}_{K}$ so that if $\hat{t}-\epsilon\left(\bar{t}-t_{0}\right)$ is large enough for $t_{0}$ then so is $\underline{t}_{K}$, a contradiction. To prove the second statement, notice that if we do not stop Algorithm 2 after finitely many steps, we get a weakly increasing sequence $\left(\underline{t}_{k}\right)$ that is bounded above (by $\bar{t}$ ) and a weakly decreasing sequence $\left(\bar{t}_{k}\right)$ that is bounded below (by $t_{0}$ ) and hence both these sequences converge. Also, for every $k$, $\underline{t}_{k} \leq \bar{t}_{k}$ and $\bar{t}_{k}-\underline{t}_{k}=\frac{1}{2^{k}}\left(\bar{t}-t_{0}\right)$, so that both the sequences converge to the same point, say $t^{*}$. Suppose there is a point $\hat{t}$ in the line segment from $t_{0}$ to $\bar{t}$ that is large enough for $t_{0}$. If $\hat{t}<t^{*}$ then for some $k$ sufficiently large, $\hat{t}<\underline{t}_{k}$ so that $\underline{t}_{k}$ is large enough for $t_{0}$, a contradiction. Therefore, $t^{*} \leq \hat{t}$ and $t^{*}$ is a lower bound. The convergence of $\bar{t}_{k}$ to $t^{*}$ implies that $t^{*}$ is a greatest lower bound.

A corollary of this proposition is that if there is a point in the line segment from $t_{0}$ to $\bar{t}$ that is the lowest element in this line segment that is large enough for $t_{0}$ then the sequences generated by Algorithm 2 converge to this point.

\footnotetext{
${ }^{3}$ This happens when $\mathcal{E}\left(t_{0}\right)$ is singleton-valued
} 


\section{Example}

As an application of Algorithm 2 we perform a comparative statics analysis of a version of Farrell and Saloner's (1985) game of network externalities. In this game there are two agents, indexed $i=1,2$. Each agent chooses a degree or probability of adoption of a new technological standard and depending on a parameter, is subsidized for technology adoption. Formally, agent $i$ chooses a number $x_{i}$ from the unit interval $[0,1]$ and for a subsidy level $t \in[0,1]$, gets a payoff of $t x_{i}$. Agent 1 's payoff is given by

$$
u_{1}\left(x_{1}, x_{2} ; t\right)=2 x_{1} x_{2}+t x_{1}+2 x_{1}\left(x_{2}-1 / 2\right)-x_{1}^{2}+\frac{1}{10}\left[\log \left(x_{1}\right)+\log \left(1-x_{1}\right)\right] .
$$

The first term reflects the fact that the gain to agent 1 is higher when agent 2 adopts more of the technology, the second term is agent 1's subsidy, the third term shows that the gain is increased more when agent 2 adopts a higher technological standard, the fourth term is a quadratic cost of technology adoption and the term in brackets prevents corner solutions. This functional form, especially the third term, helps us obtain multiple equilibria in a simple way. Agent 2's payoff is given symmetrically, that is $u_{2}\left(x_{1}, x_{2} ; t\right)=u_{1}\left(x_{2}, x_{1} ; t\right)$. A model with incomplete information will yield our example as a reduced form.

It is easy to see that this is a supermodular game parameterized by $t \in T=[0,1] \subset \Re$. Suppose the current level of the subsidy is $t_{0}=0.15$ and we want to find out if increasing the subsidy will increase the equilibrium. (Notice that an increase in the equilibrium choices of the agents implies a Pareto improvement over the old equilibrium.) We want to know what the new level of subsidy must be so that we can unambiguously conclude that both agents are better off. That is, we want to find a $\hat{t}$ large enough for $t_{0}$.

Note that all extremal equilibria are symmetric. Using Algorithm 1, we find that $\inf \mathcal{E}\left(t_{0}\right)=(0.06,0.06)$ and $\sup \mathcal{E}\left(t_{0}\right)=(0.88,0.88)$. Using Algorithm 2 with $K=5$ we obtain, at $\hat{t}=0.81, \inf \mathcal{E}(0.81)=(0.94,0.94)$. There is no significant improvement even after increasing the number of iterations to $K=20$ so we conclude that with a relatively 
small number of iterations $(K=5)$ the performance of Algorithm 2 is good. The $\mathrm{C}$ programming code for these computations is available from the authors on request.

\section{Appendix}

In this appendix we prove a lemma that is a slightly generalized version of Theorem 2.5.2 in Topkis (1998). We want to prove this version because our definition of an increasing family of correspondences is weaker than the one given in Topkis (1998). ${ }^{4}$

Lemma. Let $\left(\phi_{t}: t \in T\right)$ be an increasing family of correspondences. For every $t, t^{\prime} \in T$, if $t \preceq t^{\prime}$ then $\inf \mathcal{E}(t) \preceq \inf \mathcal{E}\left(t^{\prime}\right)$ and $\sup \mathcal{E}(t) \preceq \sup \mathcal{E}\left(t^{\prime}\right)$.

Proof: By Theorem 2.5.1 in Topkis (1998), we know that $\mathcal{E}(t)$ is a complete lattice and $\sup \mathcal{E}(t)=\sup \left\{x \in X: \phi_{t}(x) \cap[x, \sup X] \neq \emptyset\right\}$. Thus, $\sup \mathcal{E}(t) \in \phi_{t}(\sup \mathcal{E}(t))$ and hence $\sup \mathcal{E}(t) \preceq \sup \phi_{t}(\sup \mathcal{E}(t))$. As the correspondence $t \mapsto \phi_{t}(x)$ is weakly increasing, we have $\sup \phi_{t}(\sup \mathcal{E}(t)) \preceq \sup \phi_{t^{\prime}}(\sup \mathcal{E}(t)) \in \phi_{t^{\prime}}(\sup \mathcal{E}(t))$ so that $\phi_{t^{\prime}}(\sup \mathcal{E}(t)) \cap$ $[\sup \mathcal{E}(t), \sup X] \neq \emptyset$. Thus, $\sup \mathcal{E}(t) \in\left\{x \in X: \phi_{t^{\prime}}(x) \cap[x, \sup X] \neq \emptyset\right\}$ and hence $\sup \mathcal{E}(t) \preceq \sup \mathcal{E}\left(t^{\prime}\right)$. The result for $\inf \mathcal{E}(t)$ follows analogously.

\section{References}

Echenique, F. (2002): "Comparative Statics by Adaptive Dynamics and The Correspondence Principle," Econometrica, 70(2), 257-289.

Farrell, J., and G. Saloner (1985): "Standardization, compatibility and innovation," Rand Journal of Economics, 16(1), 70-83.

Lippman, S. A., J. W. Mamer, and K. F. MCCardle (1987): "Comparative Statics in Non-cooperative Games via Transfinitely Iterated Play," Journal of Economic Theory, 41(2), 288-303.

\footnotetext{
${ }^{4}$ In Topkis (1998) the section $x \mapsto \phi_{t}(x)$ is increasing in the strong set order. We only assume it increasing in the weak set order.
} 
Milgrom, P., And J. Roberts (1990): "Rationalizability, Learning and Equilibrium in Games with Strategic Complementarities," Econometrica, 58(6), 1255-1277.

Milgrom, P., and C. Shannon (1994): "Monotone Comparative Statics," Econometrica, 62(1), 157-180.

Press, W. H., B. P. Flannery, S. A. Teukolsky, and W. T. Vetterling (1989): Numerical Recipes in C. Cambridge University Press.

Sobel, M. J. (1988): "Isotone Comparative Statics in Supermodular Games," mimeo, SUNY at Stony Brooks.

Topkis, D. M. (1979): "Equilibrium Points in Nonzero-Sum n-Person Submodular Games," SIAM Journal of Control and Optimization, 17(6), 773-787. (1998): Supermodularity and Complementarity. Princeton University Press.

Vives, X. (1990): "Nash Equilibrium with Strategic Complementarities," Journal of Mathematical Economics, 19(3), 305-321.

(1999): Oligopoly Pricing. MIT Press. 